\title{
Comparison between Laparoscopy and Noninvasive Tests for the Diagnosis of Tuberculous Peritonitis
}

\author{
B. Ramana
}

Published online: 9 September 2011

(c) Société Internationale de Chirurgie 2011

In spite of the myriad imaging modalities available to the modern surgeon, the diagnosis of tuberculosis (TB) may defy everything but his clinical imagination and, perhaps, his laparoscopic vision. Herein lies the problem intertwined with the solution: while the naked eye diagnosis of TB is often obvious, it is not infrequently a vexing one. Is it cancer, pancreatitis, lymphoma, or Crohn's disease? The surgeon may ask himself many questions in different situations.

The authors of the article under consideration [1] have made an important point that needs to be stressed with respect to the diagnosis of TB: while imaging tests are usually the first line (or even the definitive one) of assessment, even the best computed tomography (CT) scanner is not able to give a reliable diagnosis in many instances. Not only is the test unable to detect tubercles, it is also dependent on the radiologist who interprets the images. In fact, if a magnified laparoscopic image of tubercle-like lesions can elicit different diagnoses among surgeons, why would the CT images of bowel thickening or adhesions be different? In terms of decision-making, one may argue that a CT scan often makes the job harder, or at least may not make things any easier.

Additionally, the most reliable test that is proof-positive for the diagnosis of TB is the culture, and most of us would agree that it is not often positive. The newer nucleic acid amplification tests seem reasonably specific, but it is the T-SPOT test (which tests for the presence of interferons) that seems to be very sensitive to TB [2]. One could also think, "would getting back to the basic, older tests help?" The ascitic fluid measurements known to every medical entrance exam candidate include ADA (adenosine deaminase) and lactase dehydrogenase $(\mathrm{LDH})$. As the authors say, these do have value, but they

B. Ramana $(\square)$

Belle Vue Clinic, 9\&10 Loudon Street, Kolkata 700017, India

e-mail: rambodoc@gmail.com are not as specific or sensitive as we would like them to be. Biopsy, of course, seems to be an attractive option to the surgeon dealing with this disease. On the slides we would see the caseating granulomas, the epithelioid cells, and the giant cells, leading us to gleefully unleash the full brunt of anti-TB poisons. Again, reality does not always follow this course.

In the paper under the spotlight, the authors have said much of the above in better and more refined language. It is unfortunate that they have had high mortality and complications following laparoscopy, likely a reflection of the patient sample they were dealing with. Their patients seem to be sicker than most. In fact, one might even wonder if laparoscopy was not too invasive a test for some of them!

The presence of atypical cells or crush artifacts in small laparoscopic biopsy fragments are some reasons for inconclusive reports.

In summary, with every test having its pitfalls, the surgeon has to find a solution that works, and one that is an acceptable basis for justifying a clinical decision. This would need proper clinical evaluation, appropriate imaging and endoscopic tests, ascitic fluid measures, and, finally, laparoscopic assessment supplemented by histopathology. Once all these are placed in clinical perspective, an answer should be reasonably plausible. If it is still not, an empirical trial of anti-TB drugs still has a role in the 21 st century, even if this sounds archaic.

\section{References}

1. Hong KD, Lee SI, Moon HY (2011) Comparison between laparoscopy and noninvasive tests for the diagnosis of tuberculous peritonitis. World J Surg 103:406-410

2. Meier T, Eulenbruch HP, Wrighton-Smith P et al (2005) Sensitivity of a new commercial enzyme-linked immuno spot assay (T SPOT-TB) for diagnosis of tuberculosis in clinical practice. Eur J Clin Microbiol Infect Dis 24:529-536 